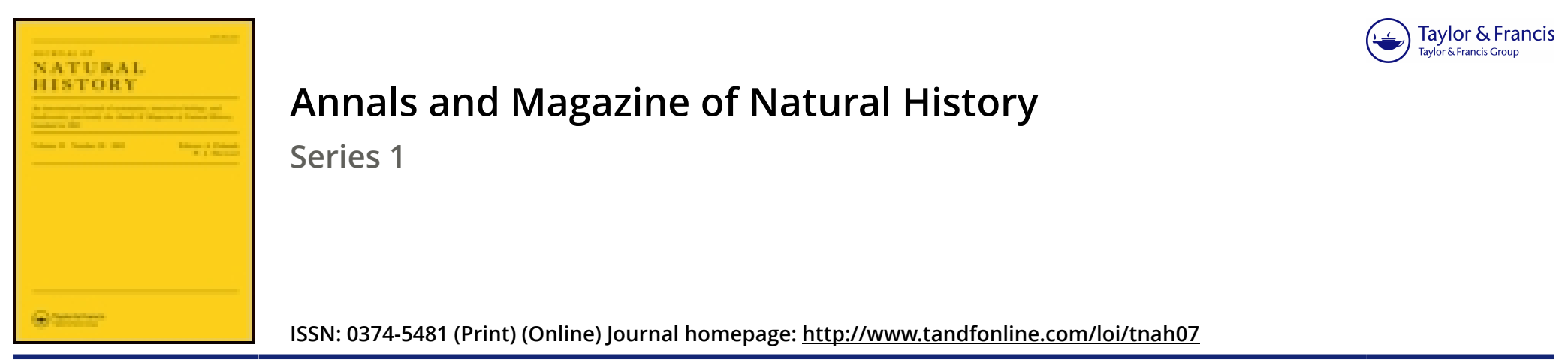

\title{
New species of volute
}

\section{J. E. Gray}

To cite this article: J. E. Gray (1846) New species of volute, Annals and Magazine of Natural History, 18:121, 431-431, DOI: 10.1080/037454809494452

To link to this article: http://dx.doi.org/10.1080/037454809494452

曲 Published online: 10 Dec 2009.

Submit your article to this journal 준

Q View related articles $\llbracket$ 
NEW SPECIES OF VOLTTE.

Voluta Sophia. Shell ovate, ventricose, white with minute red dots, and a few series of irregular red spots; last whorl ventricose, with a series of large conical tubercles behind; spire very short; the apex rather produced, rounded, crenulated.

$H a b$. North Australia, Endeavour Sound.

This species is most like Voluta cymbiola, but much more ventricose and shorter. The spire of the older specimen is covered with a callous secretion.-J. E. Gray.

\section{METEOROLOGICAL OBSERVATIONS FOR OCT. 1846.}

Chiswick.-October 1. Foggy : fine: overcast. 2. Hazy : very fine: rain, 3, 4. Very fine. 5. Cloudy. 6. Overcast : rain. 7. Fine : rain. 8. Clear : cloudy : rain. 9. Rain. 10. Boisterous : rain. 11. Fine: rain. 12. Foggy. 13. Slight rain : cloudy : clear. 14. Densely overcast: heavy rain. 15. Rain : cloudy: clear. 16. Clear : fine : rain at night. 17. Slight fog. 18. Heavy and continued rain. 19. Foggy : cloudy and fine : clear. 20. Hazy : very fine: clear. 21. Heavy rain: boisterous, 22. Clondy : boisterous. 23. Fine : clear : slight frost at night. 24. Rain : fine : rain, 25. Cloudy. 26. Foggy. 27. Hazy : fine: foggy at night. 28. Dense fog. 29. Cloudy : slight rain. 30. Hazy: overcast: clear, 31. Foggy.

Mean temperature of the month .......................... $50^{\circ} \cdot 37$

Mean temperature of Oct. 1845 .......................... $49 \cdot 96$

A verage mean temperature of Oct. for the last twenty years . $50 \cdot 43$

Average amount of rain in Oct. ............................ 2.58 inches.

Boston.-Oct. 1. Fine. 2. Cloudy and foggy : rain A.m. and P.M. 3. Cloudy. 4. Fine. 5. Cloudy : rain P.M. 6. Fine. 7, 8. Cloudy: rain early A.M. 9. Rain : rain early A.M. : rain A.M. 10. Stormy. 11. Fine : rain P.M. 12. Cloudy: rain P.M. 15. Windy. 14. Rain : rain A.M. and P.M. 15. Fine. 16. Fine: rain early A.M. 17. Cloudy : rain early A.M. 18. Cloudy : rain A.M. and P.M 19. Fine. 20. Rain : rain early A.M. 21. Rain and stormy : rain early A.M.: rain A.M. 22, Cloudy : rain P.M. 23. Cloudy. 24. Rain : rain early A.M. 25. Fine. 26-28. Foggy. 29. Cloudy. 30, 31. Foggy.-The past month has been an extraordinary moist one.

Sandwick Manse, Orkney.-Oct. 1, 2. Cloudy : clear. 3. Bright: clear. 4, 5. Bright : hazy. 6. Cloudy : rain. 7. Clear: hazy : aurora. 8. Bright : clear. 9. Bright : raiu. 10. Rain : cloudy. 11. Bright: rain. 12. Cloudy : showers. 13. Cloudy. 14. Rain. 15. Rain : showers : aurora. 16. Bright : clear: aurora. 17. Fine: damp, 18. Bright: cloudy. 19. Rain: cloudy: aurora. 20. Bright : cloudy. 21. Cloudy : rain. 22. Bright: showers : aurora. 23. Showers : cloudy. 24. Cloudy : showers : aurora. 25. Bright : clear : aurora. 26. Frost : cloudy. 27. Frost: bright: clear: aurora. 28. Cloudy. 29, 30. Rain: drops, 31 . Showers: cloudy.

Applegarth Manse, Dumfries-shire.-Oct. 1. Rain A. m.: cleared: fine. 2, 3. Fair and fine. 4. Fair, but raw : threatening. 5. Showers. 6. Violent showers P.M. 7. Violent showers P.M. : thunder. 8. Violent showers P.M. 9. Rain all day. 10. Showers. 11. Rain P.M. 12. Showers. 13. Fine and fair. 14. Heavy rain. 15. Fair and fine. 16. Rain P.M. : thunder. 17. Slight showers. 18. Slight showers r.m. 19. Frequent showers. 20. Showers a.m.: cleared. 21 . Showers A.M. 22, 23. Fair. 24. Heavy rain, 25. Fair and clear, 26. Frost : clear. 27. Frost: cloudy. 28. Fair and fine. 29. Slight showers. 30. Rain A.M. : cleared. 31. Slight shower P.M.

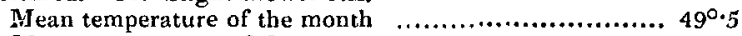

Mean temperature of Oct. 1845 ........................... $49 \cdot 6$

Mean temperature of Oct. for twenty-three years $\ldots \ldots \ldots .46 \cdot 4$

Mean rain in $0 . t$. for eighteen years ..................... $3 \frac{1}{2}$ inches. 